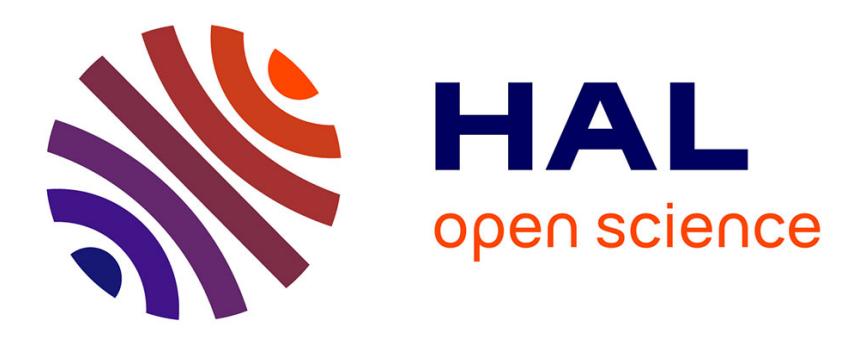

\title{
The effect of sequentiality and heterogeneity in network formation games \\ Liza Charroin
}

\section{To cite this version:}

Liza Charroin. The effect of sequentiality and heterogeneity in network formation games. 2016 . halshs-01368067

\section{HAL Id: halshs-01368067 \\ https://shs.hal.science/halshs-01368067}

Preprint submitted on 19 Sep 2016

HAL is a multi-disciplinary open access archive for the deposit and dissemination of scientific research documents, whether they are published or not. The documents may come from teaching and research institutions in France or abroad, or from public or private research centers.
L'archive ouverte pluridisciplinaire HAL, est destinée au dépôt et à la diffusion de documents scientifiques de niveau recherche, publiés ou non, émanant des établissements d'enseignement et de recherche français ou étrangers, des laboratoires publics ou privés. 
UMR 5824

93. chemin des Mouilles 69130 Ecully - France

Maison de IUniversité, Bâtiment $\mathrm{B}$ 10, rue Trefilerie 42023 Saint-Etienne cedex $02 \cdot$ France http://www.gate.cnrs.fr gate@gate.cnrs.fr

\title{
The effect of sequentiality and heterogeneity in network formation games \\ Liza Charroin
}

\begin{abstract}
:
In the benchmark model of Bala and Goyal (2000) on network formation, the equilibrium network is asymmetric and unfair as agents have different payoffs. While they are prominent in reality, asymmetric networks do not emerge in the lab mainly because of fairness concerns. We extend this model with a sequential linking decision process to ease coordination and with heterogeneous agents. Heterogeneity is introduced with the presence of a special agent who has either a higher monetary value or a different status. The equilibrium is asymmetric and unfair. Our experimental results show that thanks to sequentiality and fairness concerns, individuals coordinate on fair and efficient networks in homogeneous settings. Heterogeneity impacts the network formation process by increasing the asymmetry of networks but does not decrease the level of fairness nor efficiency.
\end{abstract}

\section{Keywords:}

Network formation, sequentiality, heterogeneity, fairness, asymmetry

JEL codes:

C72, C92, D85, Z13

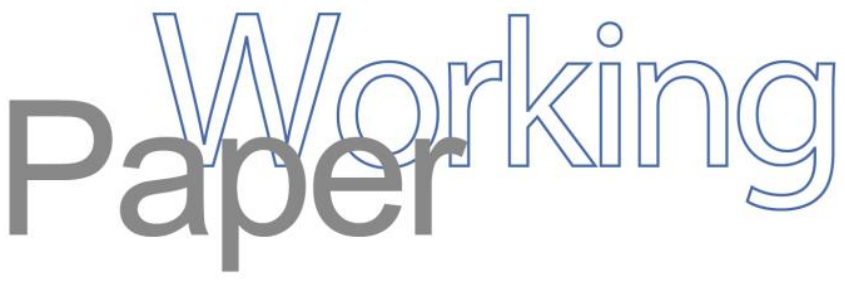




\title{
The effect of sequentiality and heterogeneity in network formation games*
}

\author{
Liza Charroin ${ }^{\dagger}$
}

September 15, 2016

\begin{abstract}
In the benchmark model of Bala and Goyal (2000) on network formation, the equilibrium network is asymmetric and unfair as agents have different payoffs. While they are prominent in reality, asymmetric networks do not emerge in the lab mainly because of fairness concerns. We extend this model with a sequential linking decision process to ease coordination and with heterogeneous agents. Heterogeneity is introduced with the presence of a special agent who has either a higher monetary value or a different status. The equilibrium is asymmetric and unfair. Our experimental results show that thanks to sequentiality and fairness concerns, individuals coordinate on fair and efficient networks in homogeneous settings. Heterogeneity impacts the network formation process by increasing the asymmetry of networks but does not decrease the level of fairness nor efficiency.
\end{abstract}

Keywords: network formation, sequentiality, heterogeneity, fairness, asymmetry.

JEL code: C72, C92, D85, Z13.

\section{Introduction}

Individuals are embedded in social and economic networks. The affiliation to a social environment influences the acts and decisions of individuals. Social networks can be created by the individuals themselves. One of the characteristics of most networks is that they are asymmetric, i.e. few nodes (individuals, firms, websites, etc.) have more links and are more central than other peripheral nodes. For example, friendship networks at school (Bramoullé and Rogers, 2009), scientific co-authorship networks (Newman, 2004) and the World Wide Web (Barabási et al., 2000) exhibit an asymmetric structure.

${ }^{*}$ This research has been supported by a grant of the French National Research Agency (ANR, FELIS Grant, ANR-14-CE28-0010-01). This work was performed within the framework of the LABEX CORTEX (ANR-11-LABX-0042) of Université de Lyon, within the program "Investissements d'Avenir" (ANR-11-IDEX007) operated by the French National Research Agency (ANR). Funding for this project was provided by a grant from Région Rhône-Alpes. I am grateful to M. Kosfeld and G. Friebel for their help and for their invitation in the Goethe University. I thank M.C. Villeval and C. Bravard for their helpful comments, Q. Thevenet for his help in programming the experiment presented in this paper and V. Theroude, C. Saucet, R. Suchon, J. Benistant and A. Solda for research assistance. I also thank B. Van Leeuwen, A. Poulsen, S. Sarangi, E. Fatas, S. Robin, F. Galeotti, A. Zylbersztejn, B. Monnery and participants at the 2015 BEE workshop in Lyon, at the 2015 ESA meeting in Heidelberg, at the 2015 CCC meeting in Norwich and at the 2016 colloquium MM in Kleinwalsertal for their interesting comments.

${ }^{\dagger}$ Univ Lyon, ENS de Lyon, GATE L-SE UMR 5824, F-69342 Lyon, France. E-mail: charroin@gate.cnrs.fr 
More particularly, the equilibrium network of the connections model of Bala and Goyal (2000) (henceforth BG) exhibits the asymmetry described above. In their two-way flow model, a link can be formed unilaterally at a cost for the agent who initiates the link, but both agents involved in the connection benefit from it. ${ }^{1}$ Direct and indirect links are equally valuable (agents benefit as well from their friends, than the friends of their friends, etc.): there is no decay. The strict Nash Equilibrium network is a Center-Sponsored Star where one agent, the central agent, creates a link with all the peripheral agents and peripheral agents remain passive. Despite the homogeneity of agents (they have similar linking cost and value to others), the equilibrium network is highly asymmetric as one agent creates all the links. Consequently, he bears all the linking costs and has a smaller payoff than the others. Falk and Kosfeld (2012) describe a network as payoff-symmetric when agents earn the same payoff and strategy-symmetric when agents create the same number of links. The Center-Sponsored Star is both payoff- and strategy-asymmetric. We define a network as fair when it exhibits payoff-symmetry and symmetric when it exhibits strategy-symmetry.

In our model, we extend the BG model without decay with two properties: heterogeneity of agents and a sequential linking decision process. First, an important characteristic of empirical networks is that individuals are usually heterogeneous per se. They have different characteristics that are more or less valuable, so creating a link with an agent may generate different benefits depending on his characteristics. The objective of this paper is to understand whether and how heterogeneity facilitates or hampers the coordination on asymmetric networks. This feature may explain why networks exhibit an asymmetric structure in real life. The heterogeneity across agents in a network has been theoretically studied (see for example Galeotti and Goyal (2010), Goyal and Joshi (2006) and Galeotti et al. (2006)). We implement heterogeneity with the introduction of a special agent, who is singled out from the others. Theoretically, we study two different settings: a homogeneous one where creating a link with any agent generates a similar value and a heterogeneous one where creating a link with the special agent generates a higher value than with any other agent.

Second, instead of taking their linking decisions simultaneously like in the model of BG, we implement a sequential linking decision process. Agents decide one after another, after observing the previous decisions. It allows a better strategic analysis capacity as decisions are taken with less uncertainty and facilitates coordination. Moreover it reflects many real-life situations as individuals rarely form links simultaneously. It is very rare that individuals have the possibility to face all the other agents and to create links at the exact same time. The formation of a network is often a dynamic process. For instance, Facebook users can observe the "friends" of a person before "adding" him as a friend. Companies often know the partnerships of another company before deciding to work with it. Co-authors can be perfectly identified on references lists of scientific papers before engaging in a joint work.

Theoretically, the introduction of sequentiality leads to a Subgame Perfect Equilibrium that is a Centrally-Sponsored Star (like in BG). More precisely based on backward induction, the last agent of the sequential process has to create a link with every other agent. This network is efficient as it maximizes the overall payoff. Note that any minimally connected network, i.e. network where $n-1$ links connect the $n$ agents, is efficient. However, the equilibrium network is both asymmetric and unfair as agents apply different strategies, between the

\footnotetext{
${ }^{1}$ In their one-way flow model, only one agent pays for the link creation and benefits from it.
} 
last agent who creates all the links and the others who remain passive. That leads to unequal payoffs: the last agent receives a very low payoff compared to the other agents. Theoretically, heterogeneity in our setting does not impact the determination of the equilibrium. Indeed, due to the no decay assumption, agents are indifferent between being directly or indirectly linked with the special agent. Consequently, the last agent creates all the links in both settings. In conclusion, our equilibrium network is a Center-Sponsored Star and is highly asymmetric and unfair like in BG.

Laboratory experiments offer a strong control for the study of network formation and their particular characteristics. The first experimentalists who tested the two-way flow model of BG are Falk and Kosfeld (2012). ${ }^{2}$ Their experiment highlights the fact that the CSS does not emerge in the lab because of the asymmetry between the central and peripheral agents and because of fairness concerns, as the central agent bears all the linking costs.

We test our model with heterogeneity and a sequential process in the lab. Based on current experimental evidences (see for example Goeree et al. (2009), Vanin (2002), Berninghaus et al. (2006), Bernasconi and Galizzi (2005) and more particularly Falk and Kosfeld (2012)), we do not expect the emergence of the equilibrium network in our experimental game due to fairness concerns. Our goal is to see whether heterogeneity impacts the fairness and the symmetry of networks. Introducing heterogeneity in the lab allows us to add a realistic feature in our experiment that can explain how asymmetry can emerge in real life. In our baseline treatment (BT), individuals are homogeneous, i.e. they have the same value to others. In our heterogeneous treatments, one individual is singled out from the others and has the status of special individual. In our experiment, we aim at analyzing which specific characteristics change the linking decisions of the normal and the special individuals. Indeed, the collaboration with a scientific author may have different values depending on the quality of the author, his reputation or his level of effort. We introduce heterogeneity in three different ways. In the exogenous treatment (EXO), the special individual is randomly selected by the computer program. In the endogenous treatment (ENDO), the selection of the special individual is based on his relative performance in a preliminary real effort task. We vary the selection of the special individual to test whether merit has an impact on linking decisions. We assume that earning the status of special individual can change his own behavior and the behavior of others towards him. In these two treatments, heterogeneity is monetary; creating a link with the special individual generates a higher benefit than with any other individual. Being directly or indirectly connected with the special individual generates the same benefit due to the no decay assumption. In the non-monetary treatment (NM), the special individual has no additional monetary value. The determination of the special individual is based on the group member's evaluation of individuals' attributes. To generate status in NM, we use the procedure of Galeotti and Zizzo (2014) described in the experimental design where individuals rank their group members based on personal attributes. This treatment is designed to investigate whether heterogeneity based on non-monetary parameters may influence the linking decisions.

We expect a strong fairness concern in the lab and so the emergence of more symmetric networks whose linking costs are shared. Our objective is to analyze whether and how heterogeneity impacts the coordination of individuals and the level of fairness and symmetry of

\footnotetext{
${ }^{2}$ The first network formation experiment based on the one-way flow model has been performed by Callander and Plott (2005). Their results show that networks happen, tend to converge to stationary configurations and that Nash equilibrium does not always predict network formation in the lab.
} 
networks compared to the baseline treatment where individuals are homogeneous.

To improve coordination on stable networks across periods, we use a sequential process. In our framework, instead of making their linking decisions simultaneously, individuals decide one after another and can observe the decisions of the previous individuals. In the experimental literature, the linking decision process is very often simultaneous. Some exceptions are described in Section 2. The reason of the introduction of this property is twofold. First, it allows individuals to act more strategically as they can observe the linking decisions of previous individuals before making their decisions. In a simultaneous process, individuals cannot anticipate the decisions of the other individuals and coordination is difficult. As we said earlier, the second reason is that it reflects many real-life situations where individuals can observe the linking decisions of others before deciding to create a link.

Goeree et al. (2009) (henceforth GOE) study the reason of the gap between theoretical and experimental findings by implementing agents' heterogeneity. The authors extend the model of BG with decay (agents benefit less from their indirect links than their direct links) and heterogeneous agents. With the introduction of value-heterogeneity where creating a link with the high-value agent generates a higher monetary benefit than with any other agent, ${ }^{3}$ the equilibrium network is not the Center-Sponsored Star but the Periphery-Sponsored Star, where each peripheral agent creates a link with the central agent (the high value agent in this case). ${ }^{4}$ Their experimental results show that the presence of a high-value individual facilitates the emergence of Periphery-Sponsored Stars. The high-value individual is more attractive and the other individuals tend to create direct links with him to fully benefit from his higher value. In their paper, heterogeneity facilitates the coordination on asymmetric networks.

The main differences between our paper and the paper of GOE are twofold. First, we implement heterogeneity in a monetary and non-monetary way and endogenously and exogenously to understand which characteristics are important to increase the attractiveness of an individual in a network formation game. In GOE, the special individual is exogenously determined and has a higher monetary value. Moreover, in our model, there is no decay. Consequently, being directly connected with the special individual is not necessary to fully benefit from him. As a consequence, we do not attempt to facilitate the emergence of star networks but our contribution is to study the effect of heterogeneity on the formation of networks. More particularly, we focus on whether heterogeneity fosters or not the coordination on asymmetric networks and whether it impacts the level of fairness. Second, we use a sequential process instead of a simultaneous one to facilitate coordination on stable networks across periods. For example, despite the strong attractiveness of the high-value individual in GOE, it takes several periods for the individuals to coordinate on the Periphery-Sponsored Star. Indeed, in the first half of the experiment, only few stars emerge.

Our experimental results show that individuals rapidly coordinate on efficient and fair networks, i.e. individuals tend to share the cost of the network formation by creating one link each. Because of this, the Sub-game Perfect Equilibrium does not emerge. However, the

\footnotetext{
${ }^{3}$ They also study cost-heterogeneity by introducing a low-cost agent who induces a lower linking cost than the others.

${ }^{4}$ A theoretical work of Galeotti et al. (2006) extended the model of BG without decay with valueheterogeneity and cost-heterogeneity. Additionally to the work of GOE, they study heterogeneity where costs and values vary across agents but also across the targeted agent for the link formation.
} 
sequential process increases the stability of networks across periods and allows the emergence of fair and efficient networks during the whole experiment. At the aggregate level, networks formed in ENDO and EXO are more asymmetric than in BT and NM. So monetary heterogeneity facilitates coordination on asymmetric networks like in GOE. Normal individuals create more links than special individuals. Moreover, in ENDO and EXO, individuals create more links with the special individual than with any other individual even if being directly or indirectly linked with the special individual leads to the same benefit. Since there is no significant difference between the attractiveness of the special individual in ENDO and EXO, merit has no impact on linking decisions. In ENDO and EXO, the first link is often formed with the special individual. Individuals are insured to be connected with him and benefit from his high value. Non-monetary heterogeneity does not impact the linking decisions. In fact, individuals are not more attracted by the special individual than by any other individual. Individuals are willing to pay to be connected with high value individuals because they guarantee a higher benefit, while they are not willing to pay to be connected with a special individual if he has only a special status and no monetary attractiveness. In summary, sequentiality facilitates coordination on efficient and fair networks but not on the equilibrium network. Monetary heterogeneity increases the asymmetry of networks as the special individual attracts more links than others.

The paper is organized as follows. In section 2, we review some papers related to asymmetric network formation and status. In section 3, we develop our theoretical model and describe the Sub-game Perfect Equilibrium. In section 4, we describe our experimental design and provide some behavioral predictions. In section 5, we develop our experimental results. Finally, section 6 concludes.

\section{Related literature}

Besides GOE, several other studies have designed environments to promote the emergence of star networks in the lab. Berninghaus et al. (2007) modify the design of the model of BG by discriminating between actively and passively reached agents. When an agent $i$ creates a link with agent $j, j$ is actively reached by $i$ while $i$ is passively reached by $j$. In their setting, an agent does not benefit from all indirect links, but only from the agents actively or passively linked with his actively reached agents. With this restriction, the Periphery-Sponsored Star (PSS) - where all peripheral agents form a link with the central agent while the central agent remains passive - is the equilibrium network and emerges frequently during the experiment. The PSS is more likely to emerge in the lab than the Center-Sponsored Star (CSS) - where the central individual forms links with all the peripheral individuals while peripheral individuals remain passive - even when individuals are homogeneous, because it is easier to coordinate on a PSS that is more symmetric as every individual but one creates one link and so participates to the linking costs. Rong and Houser (2012) keep the homogeneity assumption but designed some institutions that reflect some real-life situations to promote the emergence of star networks. Among others, they design a treatment with sequential decisions. Based on the model of Galeotti and Goyal (2010), agents have the choice of investing to acquire valuable information or obtaining it by linking to an agent who invested in information. The SPE is a PSS where the central agent is the first mover and the sole investor of the network. Surprisingly, the sequentiality property does not affect the frequency of star emergence. Based on the same kind of network formation model, Van Leeuwen et al. (2015) show that competition for status 
(being the center of the star) facilitates the emergence of the PSS, because every peripheral individual wants to be linked with this central individual. Rosenkranz and Weitzel (2012) use a different model based on public good provision and still find that agents have less difficulty to coordinate on a PSS and that this network is more stable during the experiment.

The objective of the paper is to study how heterogeneity impacts the formation of fair networks that prevail in lab experiments. Contrary to the major part of the literature (see Berninghaus et al. (2007), Rong and Houser (2012), Rosenkranz and Weitzel (2012), Van Leeuwen et al. (2015) and GOE for instance), our equilibrium network is the CSS and not the PSS. Moreover, the sequential models are designed in the context of a public good game while we purely study network formation. Our goal is not to facilitate the emergence of stars in the lab, but to facilitate the coordination on stable networks and to study the impact of heterogeneity on the formation of these networks. We find that the introduction of heterogeneity increases the asymmetry of networks.

With the introduction of heterogeneity, our work is related to the literature on status as individuals are labeled differently: normal or special individuals. For a general review on the quest for status and the effect of status with a sociological and economics approach, we refer to the survey of Weiss and Fershtman (1998) and Heffetz and Frank (2008). In this paper, we are interested in the consequences of the status of "special individual" on the linking behavior of individuals. The experimental works of Ball et al. (2001) and Ball and Eckel (1998) show that high-status individuals are better treated in a competitive environment and consequently earn a higher payoff than low-status individuals. Their status influences their own behavior and the behavior of others. Finally, Eckel and Wilson (2007) find that individuals are more influenced by high status individuals, who foster coordination. This leads to a more frequent occurrence of the efficient equilibrium. In summary, high-status individuals contribute more, but also earn more and influence the behavior of other individuals. We expect that the status of special individual will modify the preferences for linking. Additionally, the way the status is implemented can impact differently individuals' behavior. For example, in the dictator game literature, dictators who earn their status behave more selfishly than exogenously chosen dictators (see for example Hoffman and Spitzer (1985) and Cherry et al. (2002)).

Our contribution to this literature is that we study the impact of status on attractiveness and on the structure of networks in a network formation game. To our knowledge, this is the first paper studying this issue in a non-monetary manner.

\section{Theoretical model}

We extend the two-way flow model of BG without decay (Bala and Goyal, 2000) with two properties: heterogeneity and sequentiality.

\subsection{Notation}

Let $N=\{1, \ldots, n\}$ be the set of agents, with two typical agents $i$ and $j$. Agents can form links with every other agent. Let agent $i$ 's links be represented by the linking vector $g_{i}=\left(g_{i 1}, \ldots, g_{i n}\right)$ where $g_{i j} \in\{0,1\}, \forall i, j \in N$ and $g_{i i}=0, \forall i \in N$. We write $g_{i j}=1$ if agent $i$ has formed a link with $j$ and $g_{i j}=0$ otherwise. Unilateral consent is sufficient to form a link, so $g_{i j}=1$ does not imply $g_{j i}=1$. A network $g$ consists of all agents in $N$ and their links, i.e. $g=\left(g_{1}, \ldots, g_{n}\right)$. 
Let $G$ be the set of all possible networks, i.e. $g \in G$.

If $g_{i j}=1$ or $g_{j i}=1$, then $i$ and $j$ are adjacent agents, we also say that they are directly linked. The closure of $g, \bar{g}$, is defined by $\bar{g}_{i j}=\max \left\{g_{i j}, g_{j i}\right\}, \forall i, j \in N$ and $i \neq j$. A path between agent $i$ and $j$ is a sequence of distinct agents $\left(i, j_{1}, \ldots, j_{k-1}, j\right)$ where $\bar{g}_{i j_{1}}=\bar{g}_{j_{1} j_{2}}=$ $\ldots=\bar{g}_{j_{k-1} j}=1$. In the following, we say that two agents are indirectly linked if there exists a path between them but they are not adjacent. Let $N_{i}(g)$ represent the set of agents directly or indirectly linked with agent $i$. The out-degree of agent $i$ is the number of links created by $i$ : $\sum_{i \neq j} g_{i j}, \forall j \in N$. The $i n$-degree of agent $i$ is the number of agents $j \neq i$ who created a link with $i: \sum_{i \neq j} g_{j i}=\mu_{i}^{p}(g), \forall j \in N, j \neq i$. This represents the attractiveness of an agent. The degree of an agent $i$ is the sum of the in-degree and the out-degree of agent $i: \sum_{i \neq j} \bar{g}_{i j}$, $\forall j \in N$, denoted $d_{i}(g)$ in the following of the paper.

Let $g_{-i}$ be the actions taken by agents other than $i$. With a slight abuse of notation, we write $g=\left(g_{i}, g_{-i}\right), \forall i \in N$. Let $g_{i}^{-}=\left(g_{1}, \ldots, g_{i-1}\right)$ and $g_{i}^{+}=\left(g_{i+1}, \ldots, g_{n}\right)$ respectively represent the actions of agents that made their linking decisions before and after $i$.

A non-empty subset of agents $N^{\prime} \subset N$ is a component of $g$ if there exists a direct or indirect link between every two distinct members of $N^{\prime}$ but no agents in $N^{\prime}$ are directly or indirectly linked with any agent in $N \backslash N^{\prime}$. An isolated agent has a degree zero and by convention forms a component.

We now formally define some networks that will be useful in the following of the paper. The empty network is the network $g$ which contains no links. A connected network $g$ contains a unique component. A network $g$ is minimally connected if the network is connected and there is no cycle in the network. A star network is a network $g$ where an agent, say agent $i$, is adjacent with all the other agents while agents $j \neq i$ are adjacent only with $i$. We say that $i$ is the central agent of the star and agents $j \neq i$ are peripheral agents. We have different types of star networks. In the Periphery-Sponsored Star (PSS), all peripheral agents form a link with the central agent. On the contrary, in the Center-Sponsored Star (CSS) the central agent forms a link with all peripheral agents. The Mixed-Sponsored Star (MSS) is a star where both peripheral agents and the central agent form links. The PSS, the CSS and the MSS are represented in Figure 1.

\subsection{Cost, value and heterogeneity}

In our two-way flow model, one agent, say $i$, creates a link with $j$ and both agents benefit from it. A direct or indirect link between agent $i$ and $j$ generates a positive value $v_{j}$ for agent $i$ and $v_{i}$ for agent $j$. Values may differ among agents. Indeed, being linked with a particular agent may be more valuable than with another agent. As we have no decay in our model, values do not decrease with the length of the path, i.e. an agent benefits as much from direct links as indirect links. ${ }^{5}$ Two agents may be linked by more than one path. See Figure 2(iii) for example, where agent 5 and agent 1 are directly connected but also indirectly connected because $g_{12}=g_{23}=g_{34}=g_{45}=g_{51}=1$. In this case, they only benefit once from each other.

\footnotetext{
${ }^{5}$ If we relax the no decay assumption, agents with a higher value to others become more attractive, as being directly connected with them is necessary to fully benefit from their higher value. Remark that it is the case in GOE, such that agents have a strong incentive to form a link with the agent that generates a higher value.
} 
Forming a link is valuable but is also costly. We assume that the cost of forming a link $c_{i}$ is the same for every agent $\left(c_{i}=c, \forall i \in N\right)$. Indeed, we focus our interest on value-heterogeneity as we are mostly interested in the attractiveness of special agents. Moreover, GOE did not find any evidence that cost-heterogeneity facilitates the emergence of star networks.

We assume a linear payoff function where linking costs are subtracted from the benefits of linking. The payoff of agent $i$ in network $g$ is given by

$$
\pi_{i}(g)=\sum_{j \in N_{i}(g)} v_{j}-\sum_{\substack{j \in N \\ i \neq j}} g_{i j} c .
$$

\subsection{Sequential setting}

The main difference with the major part of the literature on network formation is that we use a sequential setting. Indeed, instead of creating links simultaneously, agents form links one after another in a certain order. Let $\rho=1,2, \ldots, n$ be the rule of order. The order is random. Agents are informed of their decision order in the sequential process. The first agent makes his decisions. Then the second agent observes the decisions of the previous agent and makes his decisions. The game stops when the last agent has made his decisions.

Information is complete. Agents know their own value to others and the value of the others. They also know the cost of a link and their decision order in the sequential process.

\subsection{Equilibrium, efficiency and centrality}

We are interested in the Sub-game Perfect Equilibrium (SPE) of this game. Each agent $i$ wants to maximize his payoff $\pi_{i}(g)$ that depends on the strategies of the other agents. Agent $i$ has to solve:

$$
\begin{aligned}
& \max \left\{\pi_{i}(g):\left(g_{i} \mid g_{-i}\right)\right\} \\
& \text { st. } g \in G .
\end{aligned}
$$

Using backward induction, we first study the case of the last agent.

The strategy of the last agent ( $n^{\text {th }}$ agent of the sequential process $\rho$ ) is to maximize his payoff according to the actions of the preceding agents (this set is already fixed). As there are no agents after him, he does not have to take into account what the following agents may decide. Formally, agent $n$ solves:

$$
\begin{aligned}
& \max \left\{\pi_{n}(g):\left(g_{n} \mid g_{n}^{-}\right)\right\} \\
& \text {st. } g \in G
\end{aligned}
$$

where $g_{n}^{-}$is fixed. The actions available for agent $n$ are represented by:

$$
\hat{G}_{n-1}=\left\{g \in G: \pi_{n}(g)=\max \left\{\pi_{n}(g):\left(g_{n} \mid g_{-n}\right)\right\}\right\} .
$$

His decision is the last decision of the game and determines all the payoffs.

In our setting, due to the no decay assumption, two actions may lead to the same payoff. Let $a$ and $b$ be two possible linking actions of an agent. If one action leads to a higher 
payoff, this action is preferred. However, if both actions lead to the same payoff, the agent is indifferent between these two actions. Concretely, if two agents $i$ and $j$ form a component and that a third agent $k$ wants to create a link with this component, he is indifferent between creating a link with $i$ or $j$. In order to have uniqueness of equilibrium, we define a tie-breaking rule. Let suppose that we have two possible linking actions $a=$ and $b=$ that lead to the same payoff for the agent. We look at the lexicographical order $\left(a_{1}, a_{2}\right)=a \succ\left(b_{1}, b_{2}\right)=b$ if $a_{1}>b_{1}$ or if $a_{1}=b_{1}$ and $a_{2}>b_{2}$. In our setting, if an agent has more than one action that leads to the same payoff, he chooses the lexicographically greater linking vector. Concretely, due to our tie-breaking rule, he chooses to link with the agents who play earlier in the sequential process $\rho$. Formally, the restricted feasible actions for the agent are:

$$
G_{n-1}=\left\{g \in \hat{G}_{n-1}: \pi_{n}(g)=\pi_{n}\left(g^{\prime}\right) \Longrightarrow g_{n} \succ g_{n}^{\prime}, \forall g^{\prime} \in \hat{G}_{n-1}\right\} .
$$

The second to last agent knows the action taken by the agents before him. However, the last agent has not already chosen his action. When the second to last agent takes his decision this will define the actions that the last agent should take to maximize his payoff. Formally, agent $n-1$ solves:

$$
\begin{aligned}
& \max \left\{\pi_{n-1}(g):\left(g_{n-1} \mid g_{n-1}^{-}\right)\right\} \\
& \text {st. } g \in G_{n-1} .
\end{aligned}
$$

Then we generalize the problem at the $i$-level. Formally, the potential actions are given by:

$$
\hat{G}_{i}=\left\{\hat{g} \in G_{i+1}: \pi_{i+1}(\hat{g})=\max \left\{\pi_{i+1}(g):\left(g_{i+1} \mid g_{i+1}^{-}\right)\right\}\right\} .
$$

We restrict the feasible actions with our tie-breaking rule:

$$
G_{i}=\left\{g \in \hat{G}_{i}: \pi_{i+1}(g)=\pi_{i+1}\left(g^{\prime}\right) \Longrightarrow g_{i+1} \succ g_{i+1}^{\prime}, \forall g^{\prime} \in \hat{G}_{i}\right\} .
$$

The maximization problem of agent $i$ can be written as:

$$
\begin{aligned}
& \max \left\{\pi_{i}(g):\left(g_{i} \mid g_{i}^{-}\right)\right\} \\
& \text {st. } g \in G_{i} .
\end{aligned}
$$

The Subgame Perfect Equilibrium is a network where each agent $i \in N$ solves the maximization problem given in equation 2 .

We also present the concept of efficiency. Traditionally, we assess the welfare of a network with the sum of individual payoffs:

$$
W(g)=\sum_{i \in N} \pi_{i}(g)
$$

A network is efficient if $W(g) \geq W\left(g^{\prime}\right), \forall g^{\prime} \in G$ (see Figure 2(i) and Figure 2(ii) for examples of efficient networks). We measure the level of efficiency of network $g$ with the ratio $W(g) / W\left(g^{*}\right)$, where $g^{*}$ is an efficient network.

A network is fair if payoffs across agents are similar. We measure the level of fairness of network $g, f(g) \in[0,1]$, with the ratio between the lowest and the highest payoffs. Formally:

$$
f(g)=\min \pi_{i}(g) / \max \pi_{j}(g), \forall i, j \in N, i \neq j .
$$

When $f(g)=1$, the network is fair (see Figure 2(iii) for an example of fair network). 
Finally, to measure the symmetry of a network, we use the standard definition of degree centrality of a network (Freeman, 1978). The degree centrality of a network can be computed by comparing the degree of the most central agent (agent who has the highest degree) with the degree of all the other agents of the network. Formally, we have:

$$
S(g)=\frac{\sum_{i \in N}\left[\max _{j \in N} d_{j}(g)-d_{i}(g)\right]}{(n-1)(n-2)}, \forall i, j \in N, i \neq j .
$$

$S(g) \in\{0,1\}$, where 0 represents the least central network where each agent has the same degree and 1 represents the most central network: the star-network. The denominator represents the most central network: the star network where one agent has a degree of $n-1$ and $n-1$ agents have a degree of 1 . This simple measure allows us to evaluate if the network is asymmetric and not the influence of an agent for example. An increasing centrality index reflects a network that is closer to a star network than a less central network. Moreover, this centrality measure of networks has been used in GOE. However, as links are formed unilaterally, we define an in-degree centrality measure that takes the in-degree of agents as variable instead of the degree to assess whether there is an agent that is more attractive than others within the network. The in-degree centrality of a network is computed by comparing the in-degree of the most central agent with the in-degree of all the other agents of the network. Formally, we have:

$$
S^{p}(g)=\frac{\sum_{i \in N}\left[\max _{j \in N} \mu_{j}^{p}(g)-\mu_{i}^{p}(g)\right]}{(n-1)(n-1)}, \forall i, j \in N, i \neq j .
$$

The denominator represents the most central network: the PSS where one agent has an indegree of $n-1$ and $n-1$ agents have an in-degree of 0 . Like the degree centrality measure, our in-degree centrality measure lies between 0 and 1 where 0 represents a network where every agent has an equal in-degree and 1 represents the PSS. Note that the denominators of $S(g)$ and $S^{p}(g)$ are different. The denominator of $S^{p}(g)$ can be higher than the denominator of $S(g)$ as an agent can have a null in-degree and still be part of the network if he creates a link but no one creates a link with him.

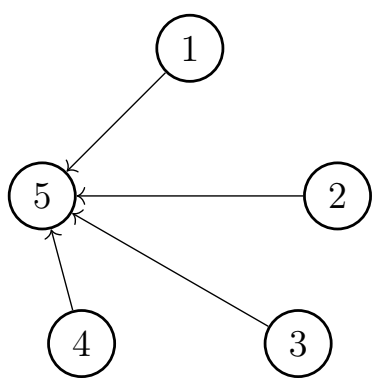

(i) PSS, $S^{p}(g)=S(g)=1$

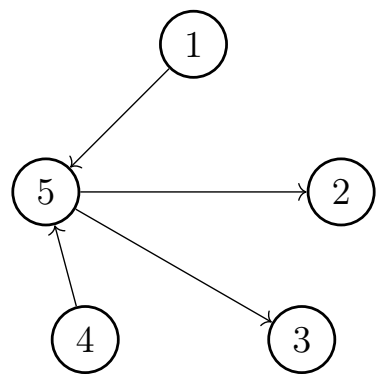

(ii) MSS, $S(g)=1$ and $S^{p}(g)=3 / 8$

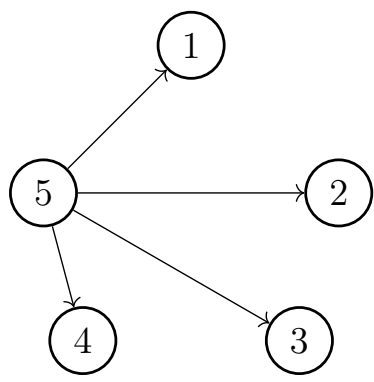

(iii) CSS, $S(g)=1$ and $S^{p}(g)=1 / 16$

Figure 1: Examples of networks with their centrality measures, labels represent the rule of order $\rho$.

\subsection{Theoretical results}

We present two major results. The first result determines the efficient networks. The second result explains that due to sequentiality, the SPE is a CSS where the last agent creates a link 
with every other agent. Consequently, his payoff is always smaller than the payoff of the other agents. The SPE is the same in the homogeneous setting and in the heterogeneous setting due to the no decay assumption. The main determinant of our model is sequentiality.

First, note that a network should always have no more than $n-1$ links if it creates a unique component.

Proposition 1 Let the payoff be given by (1) and $c<v_{i}, \forall i \in N$. A network is a non-empty efficient network if and only if it is minimally connected.

Even if they are linked by more than one path, agents only benefit once from being linked with an agent and due to the no decay assumption, all the connected networks lead to the same overall benefit. The benefits are maximized as every agent benefits from each other agent in a connected network. By definition, the minimally connected networks minimize the costs of network formation. Consequently, they maximize the overall payoff. Note that $n-1$ links are sufficient for $n$ agents to benefit from every agent and to minimize the costs of network formation.

We now study the SPE of our game in both the homogeneous and heterogeneous settings where an agent has a higher value than others.

Proposition 2 We assume $c<v_{i}, \forall i \in N$. Let the payoff be given by (1). The SPE is the CSS with the last agent as central agent.

We begin the proof with the homogeneous setting, where $v_{i}=v, \forall i \in N$ to give the intuition of the general result. Agents 1 to $n-1$ in the rule of order $\rho$ know that the last agent can create enough links to connect all the agents and so can maximize the overall benefit. They also know that he will maximize his payoff when it will be his turn. Consequently agents 1 to $n-1$ remain passive. When the last agent faces an empty network, he has no choice but maximizing the number of links to maximize his payoff. Due to the sequential process, the last agent creates all the links of the network. As there is no decay and the benefit of a link is always higher than the cost of a link, the SPE is a CSS with the last agent as central agent. The order of agents in the sequential process defines their strategy. As only the initiator of a link pays the linking cost, the last agent has a smaller payoff than peripheral agents. Formally, $\pi_{n}(g)=(n-1)(v-c)$ while $\pi_{i}(g)=(n-1) v, \forall i \in N \backslash\{n\}$.

Now, we study the case where one agent generates a higher value than others. We assume $c<v_{i}, \forall i \in N$ such that each agent can create $n-1$ links and keeps a positive payoff. However, links are costly and due to the no decay assumption, there is no need to be directly connected with the special agent to fully benefit from him. Heterogeneity does not change the structure of the equilibrium network. Indeed, because of the no decay assumption, the SPE remains the CSS with the last agent of the sequential process as central agent. Regardless of whether the last agent is a normal or a special agent, he can create all the links and so the others remain passive. $^{6}$

\footnotetext{
${ }^{6}$ It is easy to generalize our results for the cases where $c>v_{i}$ for some $i$ or for all $i$. Even if creating a link is more costly than it is beneficial, agents also benefit from their indirect links. So by creating one link, if other agents create links, they can also benefit from indirect links. Because of the sequentiality of the process, the last agent always has to create the maximum number of links he can, given the parameters. And the preceding agent needs to create the links that the last agent cannot create, etc. For example if $c>v_{j}$ and $4 v_{j}>c>3 v_{j}$
} 
The same types of network structures - minimally connected networks and star-networks - emerge in the model of BG. However, due to sequentiality one agent is always the center of the CSS at the equilibrium: the last agent. Additionally, because of the no decay assumption the introduction of a special agent does not affect the determination of the SPE. The SPE is represented in Figure 2(ii).

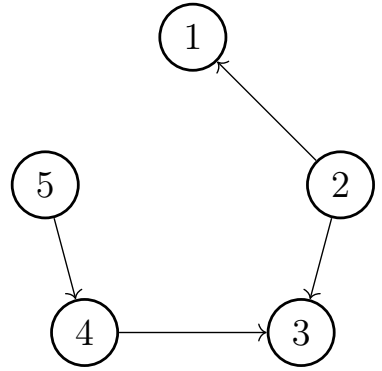

(i) Example of an efficient network

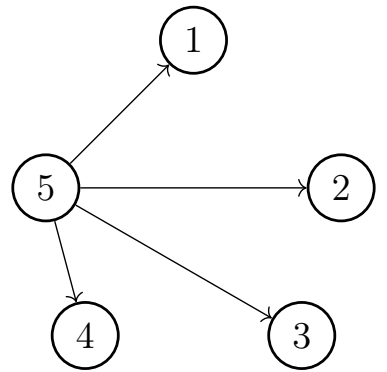

(ii) SPE, an efficient network

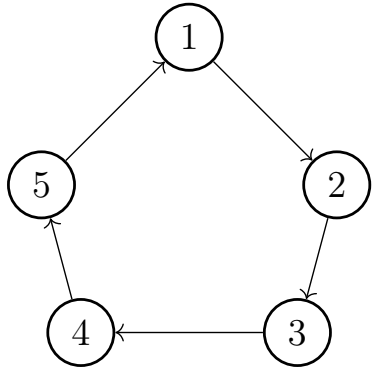

(iii) Example of an inefficient and fair network

Figure 2: Examples of networks, labels represent the rule of order $\rho$.

\subsection{Fairness}

Fairness could influence the linking decisions of agents, as it leads to unequal payoffs because the last agent bears all the linking costs. We use the model of Fehr and Schmidt (1999) to capture the impact of inequality aversion in our model. In Fehr and Schmidt's model, additionally to their own monetary payoff, agents are concerned with disadvantageous inequality (envy) and with advantageous inequality (guilt), respectively denoted by the coefficients $\alpha_{i}$ and $\beta_{i}$ for agent $i$ where $0 \leq \beta_{i}<1$ and $\beta_{i} \leq \alpha_{i}$. The utility of agent $i$ is given by:

$$
u_{i}\left(\pi_{i}(g)\right)=\pi_{i}(g)-\frac{\alpha_{i}}{n-1} \sum_{\substack{j \in N \\ j \neq i}} \max \left\{\pi_{j}(g)-\pi_{i}(g), 0\right\}-\frac{\beta_{i}}{n-1} \sum_{\substack{j \in N \\ j \neq i}} \max \left\{\pi_{i}(g)-\pi_{j}(g), 0\right\}
$$

Inequalities between agents decrease the utility of agent $i$. We take the example of a group of 5 agents where one is the special agent and four are normal agents to be consistent with our experimental design. If no link has been created until it is his turn, the last agent can decide to create between 0 and 4 links. If he is not inequality averse, the last agent should create 4 links to create a unique component of 5 agents and maximize his own payoff (as well as the overall payoff). However, if his envy coefficient is high enough, i.e. if $\alpha_{n}>0.4$ in the homogeneous setting, $\alpha_{n}>0.571$ in the monetary heterogeneous setting where the last agent is not the special agent and $\alpha_{n}>0.348$ where the last agent is the special agent, the last agent will prefer not to create any link and have a null payoff. ${ }^{7}$ The emotional cost of creating these links is higher than his monetary payoff.

$\forall j \in N$, the SPE is a network where each agent (except the first agent) creates a link with the first agent of the sequential process $\rho$.

${ }^{7}$ We compute $\alpha$ thanks to the payoff function (1) and the utility function (3) with $n=5, c=25, v_{j}=35$ if $j$ is a not a special agent and $v_{j}=50$ if $j$ is the special agent. Based on experimental results, Fehr and Schmidt (1999) depict natural levels of inequality aversion; $0 \leq \alpha_{i} \leq 4$ and $0 \leq \beta_{i} \leq 0.6$ are considered as natural levels. 
Similarly, agents in decision order 1, 2, 3 and 4 in the sequential process may not be purely selfish. Their guilt coefficient pushes them to create some links if they anticipate (or observe) that others will (or have) create(d) links, but their envy coefficient retains them from creating too many links, otherwise the others would earn more than them. Consequently, fairness concerns should encourage agents to form symmetric and fair networks.

\section{Experimental design}

We design an experiment to test our theoretical model.

\section{$4.1 \quad$ Treatments}

Our experiment uses a between-subject design. In each treatment, we randomly form fixed groups of 5 individuals. We first present the baseline treatment (BT) where individuals characteristics (values and costs) are homogeneous. After being assigned to a group of five individuals, participants are informed about the cost and the value of a link. We fix $c=25$ and $v=35$. Then, we allocate randomly the decision order in the sequential process and we randomly allocate a Greek symbol to each individual to avoid focal points. Letters or numbers to identify individuals can influence their decisions. For example, A may be more likely to form a link with $\mathrm{B}$ who may be more likely to form a link with $\mathrm{C}$, etc. The first individual of the sequential process begins. At this stage, no links have been formed yet. He can decide to create $0,1,2,3$ or 4 links. Individual 2 observes the linking decisions of individual 1 and can also create $0,1,2,3$ or 4 links. Individuals can create a link with all the other individuals, including those who have already played. Individuals 3, 4 and 5's decision rules are similar. Once individual 5 has made his decisions, the period is over and another period can start. The game lasts 10 periods. We randomly change the order of the sequential process at the beginning of every period, such that individuals do not remain in the same order during the whole experiment. Additionally, we reallocate the symbols to avoid reciprocity within the network from one period to another.

At the end of each period, we display a map of the network with the number of links initiated by each individual and we give them a feedback on their payoffs for the period. At the end of the experiment, we randomly draw one period to determine their earnings for the experiment.

To study the effect of monetary heterogeneity, in each group of five individuals, four are normal individuals and one is the special individual with a higher value. Creating a link with this special individual leads to a higher benefit. We design two treatments with different ways to implement the special individual. In the endogenous treatment (ENDO), the special individual is selected based on his relative performance during a preliminary task. After the formation of groups and before the network formation game, individuals compete in a real effort task: the slider task (Gill and Prowse, 2012). ${ }^{8}$ Then we tell individuals if they were the best performer of their group or not. The best performer is singled out from the other individuals in the network formation game. In the exogenous treatment (EXO), the special

\footnotetext{
${ }^{8}$ The screen displays 20 sliders. All the sliders are positioned on 0 . Individuals can move the sliders between 0 and 100 as many times as they want. The objective of the task is to position the maximum number of sliders at 50 in two minutes. When individuals put all the sliders displayed on their screen at 50, a new page appears with other sliders, so that they can continue the task.
} 
individual is randomly drawn after the formation of the groups.

The information about the type of each individual is public information within the group. The network formation game has the same setting than in the baseline treatment. The only difference is that the special individual has a higher value to others. The parameters are the following: the cost is unchanged, $c=25$, but forming a link with a special individual generates a gain of 50 points instead of 35 for the other individuals.

In the non-monetary heterogeneous treatment (NM), individuals have the same monetary value but one individual is singled out from the others. Like in ENDO, we want this difference to be endogenous. We use the procedure of Galeotti and Zizzo (2014). After the formation of groups, individuals have to fill a form with personal information ${ }^{9}$ to create their "profile". Then individuals can observe and rank the profile of their group members from the most preferred to the least preferred profile. Their profile is only associated with a letter such that it remains anonymous. The computer allocates a certain number of points to each individual corresponding to the ranking made by his group members. Being ranked first by another individual gives four points, being ranked second gives three points, etc. We compute the total number of points received by each individual. This ranking procedure is a Borda count. The individual with the highest number of points is the special individual of the group in the rest of the experiment. Our purpose for this treatment is to compare our results with the two monetary heterogeneous treatments and with the baseline treatment. In the three heterogeneous treatments, the special individual is graphically represented on the screen by a different color (See Figure 6 and 7 in Appendix).

\subsection{Behavioral hypotheses}

We now make some behavioral hypotheses as payoff maximization is not the sole determinant of economic decisions. Many other variables may have an influence.

There are two sorts of limitations for payoff maximization that may prevent the emergence of the SPE. The first one is cognitive. Participants can have difficulties to compute the best strategies to maximize their payoff. Understanding that remaining passive is the best strategy is not obvious. There is also a debate in network economics about the farsightedness of individuals. ${ }^{10}$ They can take rational decisions in the short term that are not beneficial in the future. In our sequential setting, participants may find it difficult to guess what could be the decisions of their subsequent group members.

Second, additionally to their self-interest, individuals may be concerned with social motives and can be inequality averse. In our setting, the benchmark equilibrium is unfair, as the last individual has to form all the links and so bears all the costs of network formation. As we showed with the model of Fehr and Schmidt (1999), the SPE may not emerge if the last individual is inequality averse and/or if the other individuals are not purely payoff-maximizers. However, we can also study our model in a dynamic way. Indeed, the network formation game is repeated 10 times and the order of decision in the sequential process changes at each period.

\footnotetext{
${ }^{9}$ The criteria are the following: gender, marital status, name of school or employer, sport, favorite dish, favorite music style, frequency of Facebook use, favorite journal and favorite movie.

${ }^{10}$ See Mantovani et al. (2011), Van Dolder and Buskens (2014) for experimental works and Morbitzer et al. (2014) and Herings et al. (2009) for theoretical works.
} 
The SPE may still emerge as the last individual of the sequential process is chosen randomly from one period to another. Indeed, a rotation is induced by the computer program. This rotation has been witnessed in the lab. In Falk and Kosfeld (2012), individuals make the decision in a pre-play communication to change position inside the network to equalize payoffs in the overall experiment. In Berninghaus et al. (2004), thanks to a continuous setting, individuals can change position in the network not to remain in the position of central individual too long. Each individual sacrifices one after another. Moreover, in our setting, individuals do not need to coordinate on which individual is going to create the links for the period as the computer program assigns the order of decision. However, limited farsightedness and coordination complexity make this rotation process complicated without pre-play communication and individuals are uncertain about the behavior of the individuals in the next periods. These limitations lead to our first hypothesis.

Hypothesis 1 SPE are unlikely to emerge, regardless of the treatment, due to fairness considerations.

We now make some predictions about heterogeneity. Theoretically, monetary and nonmonetary heterogeneity does not change the equilibrium. Sequentiality is the main determinant. However, it may have an impact experimentally. Sequentiality should increase the coordination of individuals thanks to the observation of previous decisions while heterogeneity can modify their linking decisions, like the level of fairness or the symmetry of the network. We expect more asymmetry with the presence of a special individual due to their special status and their attractiveness.

Hypothesis 2 Networks in ENDO, EXO and NM are more asymmetric than in BT due to heterogeneity.

We now make more precise hypotheses concerning the attractiveness of special individuals. We expect the attractiveness of the special individual in ENDO and EXO due to their monetary value. For the case of the special individual in NM, two reactions are possible. He deserves his status because he is elected and preferred by the others. However, being the sole individual of the group having this status may increase the social distance between him and the normal individuals. ${ }^{11}$ Moreover, the special individual is the preferred individual of the group, but he has not been necessary ranked first by all the individuals and they do not know whether the special individual is the one they ranked first. Our setting creates a normative social distance as individuals have different status in the experiment. This social distance may create a negative feeling towards the special individual and leads to his discrimination by the 4 normal individuals. In fact, they could be less willing to create links with the special individual as he has no additional monetary value and is in a different group than them. This feeling may be reinforced by the fact that normal individuals have not been selected by their group and feel envious.

\footnotetext{
${ }^{11}$ In the sociological literature, the social distance is defined as the distance between individuals who have different social characteristics. Three definitions have been described by Karakayali (2009). At the origins, it has been defined as the feelings (positive or negative) towards members of another group (individuals from a foreign country). This is called an affective social distance. The normative social distance describes the fact that norms express distinctions between individuals. Finally, the interactive social distance is strongly related to networks studies. Indeed, when two individuals from different groups interact, they are considered as socially close.
} 


\section{Hypothesis 3 - The special individual is more attractive in the three heterogeneous treat-} ments.

- The special individual in NM is less attractive than in ENDO and EXO because in spite of his status he has no additional monetary value.

- The special individual is more attractive in ENDO than in EXO due to merit.

\subsection{Procedures}

The experimental sessions were conducted at GATE-LAB, Lyon, France. We ran two sessions of each treatment with 20 individuals in each session. In total we had 160 participants. $57 \%$ of them are female and $84 \%$ are undergraduate students, $10 \%$ are employees and $6 \%$ are unemployed. At the beginning of the experiment, we randomly allocated each participant to a computer. Instructions were read out loud and the experimentalists checked the individuals' understanding and answered additional questions in private. When participants indicated that there were no more questions, the experiment started.

Before the network formation part, we elicited risk attitudes using the procedure of Gneezy and Potters (1997). ${ }^{12}$ At the end of the experiment, individuals participated in the Social Value Orientation test (Murphy et al., 2011) to evaluate their social preferences. ${ }^{13}$

Participants were recruited online through H-root (Bock et al., 2014). Each session lasted 75 minutes. Participants earned on average 15.26 Euros (SD 1.96).

\section{$5 \quad$ Experimental results}

First, we present our results concerning the type of networks groups coordinated on. Then we consider more precisely the special and normal individuals' behavior. Table 1 summarizes the most important variables at the network level.

\subsection{Networks emergence}

Result 1 (SPE, efficiency and fairness) No SPE emerged. Agents managed to coordinate on efficient and fair networks in all treatments.

Our first observation is that no SPE, the CSS with the last individual as central agent, emerged whatever the treatment. This result supports Hypothesis 1. However, we observe that $80.94 \%$ of networks are efficient, i.e. groups maximized the overall payoff by creating minimally connected networks. Moreover, on average networks have a level of efficiency, calculated as the ratio between the overall payoff and the maximum overall payoff, of $93 \%$. As

\footnotetext{
${ }^{12}$ Individuals have to choose the amount of points between 0 and 100 points that they want to invest in a risky investment. There is a $50 \%$ chance that the investment succeeds. If it is a success, the investment is multiplied by 2.5. However, if it is not a success, the investment is lost. Individuals keep for them the points they did not invest. In our experiment, $78.75 \%$ are risk-averse while the rest is risk-neutral or risk-lover.

${ }^{13}$ In this test, they have to allocate a certain amount of points between themselves and another individual. There are nine possible allocations for each of the six decisions to make. Afterward, agents can be ranked in four different categories: altruistic, competitive, individualist and pro-social. In our experiment, $35 \%$ of the agents are pro-social, $64.38 \%$ are individualist, less than $1 \%$ are competitive and none of them is altruistic. This kind of distribution is standard in the experimental economics literature.
} 
Table 1: Summary statistics at the network level

\begin{tabular}{|c|c|c|c|c|}
\hline Treatment & BT & ENDO & EXO & NM \\
\hline \hline Efficiency (\%) & $93.74(14.84)$ & $93.50(15.69)$ & $93.14(16.96)$ & $93.86(15.58)$ \\
\hline Fairness (\%) & $69.28(29.12)$ & $71.66(31.03)$ & $73.29(32.94)$ & $69.91(28.23)$ \\
\hline Centrality & $0.32(0.21)$ & $0.35(0.26)$ & $0.41(0.24)$ & $0.32(0.24)$ \\
\hline Indegree centrality & $0.27(0.16)$ & $0.34(0.21)$ & $0.39(0.22)$ & $0.27(0.20)$ \\
\hline
\end{tabular}

Note: The numbers represent the means by treatment and the standard deviations are given in parentheses. Efficiency (\%) represents the average efficiency level of networks, calculated as the ratio between the overall payoff of the network and the maximum overall payoff. Fairness (\%) shows the average fairness level of networks, calculated as the ratio between the lowest payoff and the highest payoff in the network. Centrality represents the average level of degree centrality of networks and Indegree centrality represents the average level of in-degree centrality of networks. The formal definitions are given in Section 3.4. The statistical tests to measure the differences across treatments are given in the body of the text.

shown in Table 1, the efficiency levels are very close across treatments. 80 networks are formed in each treatment, 10 per group. We have 8 groups per treatment, so 8 independent observations. There is no significant difference of efficiency levels across treatments (Mann-Whitney test). For each non-parametric tests on networks emergence, we compare the means by network across treatments and the tests are two-tailed. The sequential process allows individuals to form the number of links that maximizes the overall payoff. In the literature on network formation with a simultaneous process, groups tend to over-connect or under-connect as they cannot anticipate the links created by their group members, ${ }^{14}$ which leads to welfare losses.

One feature can explain the fact that no SPE emerge: the asymmetry of the CSS and so the inequalities in payoffs. Individuals tend to create only one link per period to form fair networks. In fact, $71,63 \%$ of the decisions are to create exactly one link and only $2,82 \%$ of the decisions are to create more than one link. As a consequence, the level of fairness is very high in all treatments. On average, the level of fairness is of 71\%: 69.28 in BT, 71.66 in ENDO, 73.28 in EXO and 69.91 in NM. Networks are significantly more fair in ENDO and EXO than in BT and NM $(p<0.001$ between EXO and BT and between EXO and NM, $p=0.01$ between ENDO and NM and $p=0.023$ between ENDO and BT). Surprisingly, payoffs are better equalized between individuals in ENDO and EXO than in the two other treatments. The emergence of efficient and fair networks is very stable across periods. Groups managed to maintain high levels of fairness and efficiency from period 1 to period 10 as we can see in Figure 3.

Another phenomenon can confirm that fairness is an important concern for agents. 37 individuals ( $23 \%$ of the individuals) have been excluded at least once during the network formation process in total regardless of the treatment. Some have been excluded more than once, exclusion happened 45 times: 11 times in BT, 10 times in ENDO and NM and 14 times in EXO. It affected the formation of 40 networks, namely $12.5 \%$ of the networks. These individuals did not create any link when it was their turn to decide and the subsequent individuals punished them by not creating links with them. The exclusion of these non-collaborative individuals leads to welfare losses. This exclusion is costly for the group as creating a link

\footnotetext{
${ }^{14}$ See for example Buechel and Hellmann (2012) and Callander and Plott (2005) for experimental evidence and Morrill (2011) for a theoretical work.
} 
Figure 3: Average efficiency and fairness levels of networks across periods

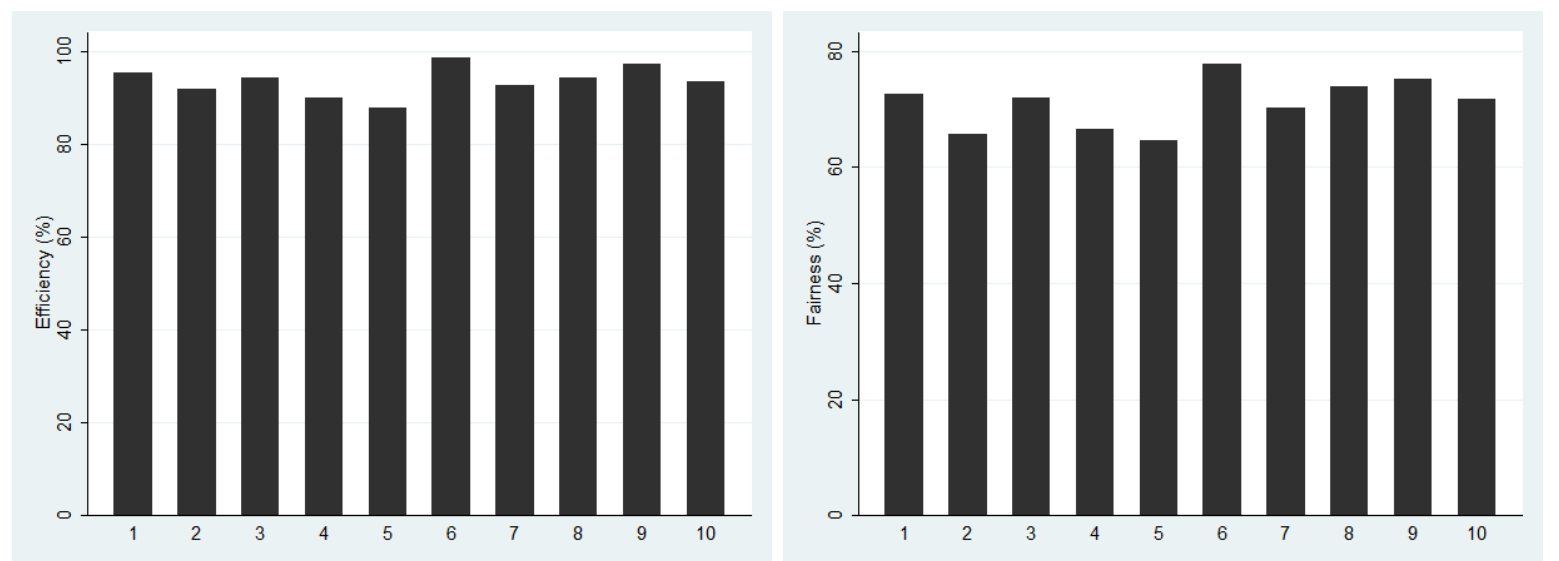

Note: Efficiency (\%) represents the average level of efficiency of networks, calculated as the ratio between the overall payoff of the network and the maximum overall payoff. Fairness (\%) represents the average level of fairness of networks, calculated as the ratio between the lowest payoff and the highest payoff in the network.

is always valuable and not excluding this individual would increase the overall payoff. For instance, if one individual is isolated and the four others are minimally connected, it leads to a welfare loss of 255 points (17 Euro) for the group compared to an efficient network where the five individuals are minimally connected.

Exclusion is sub-optimal for the whole group as one period is randomly selected for the payment. But individuals who have been excluded tend to cooperate more in the subsequent periods of the experiment. Only 7 individuals have been excluded more than once. Individuals who have been excluded once create a link in $80 \%$ of the cases in the subsequent rounds, while individuals who have never been isolated create a link in $74 \%$ of the cases. The difference is significant, $p=0.049$. It seems that in the experiment, groups implement the norm of creating each one link, so that every one participates. In fact, exclusion reinforces the coordination of individuals on fair networks. Riedl and Ule (2002) have already identified this phenomenon in their experiment on network formation and collaboration. Indeed, individuals who are able to exclude defectors from the group at a cost can maintain high levels of coordination.

Groups formed fair and efficient networks. We now turn to the analysis of whether heterogeneity impacts the centrality of networks.

Result 2 (Centrality) Networks formed in ENDO and EXO are more asymmetric than in $N M$ and BT. The centrality level of networks in NM and BT is similar.

This result partly supports Hypothesis 2. Heterogeneity increases the asymmetry of networks when heterogeneity is monetary but not when heterogeneity is non-monetary. Networks are more central in EXO than in the other treatments $(p=0.012$ between EXO and BT, $p=0.036$ between EXO and ENDO and $p=0.006$ between EXO and NM.). Centrality is also higher in ENDO than in BT or NM, but not significantly. This centrality measure is not sufficient to analyze the asymmetry of networks as links are unilaterally formed and that the attractiveness of the special agent is important in our setting. Figure 4 shows the level of in-degree centrality of networks across treatments. We notice that the level of in-degree centrality is significantly higher in ENDO and EXO compared to BT and NM ( $p=0.053$ between ENDO and BT, 
Figure 4: In-degree centrality of networks

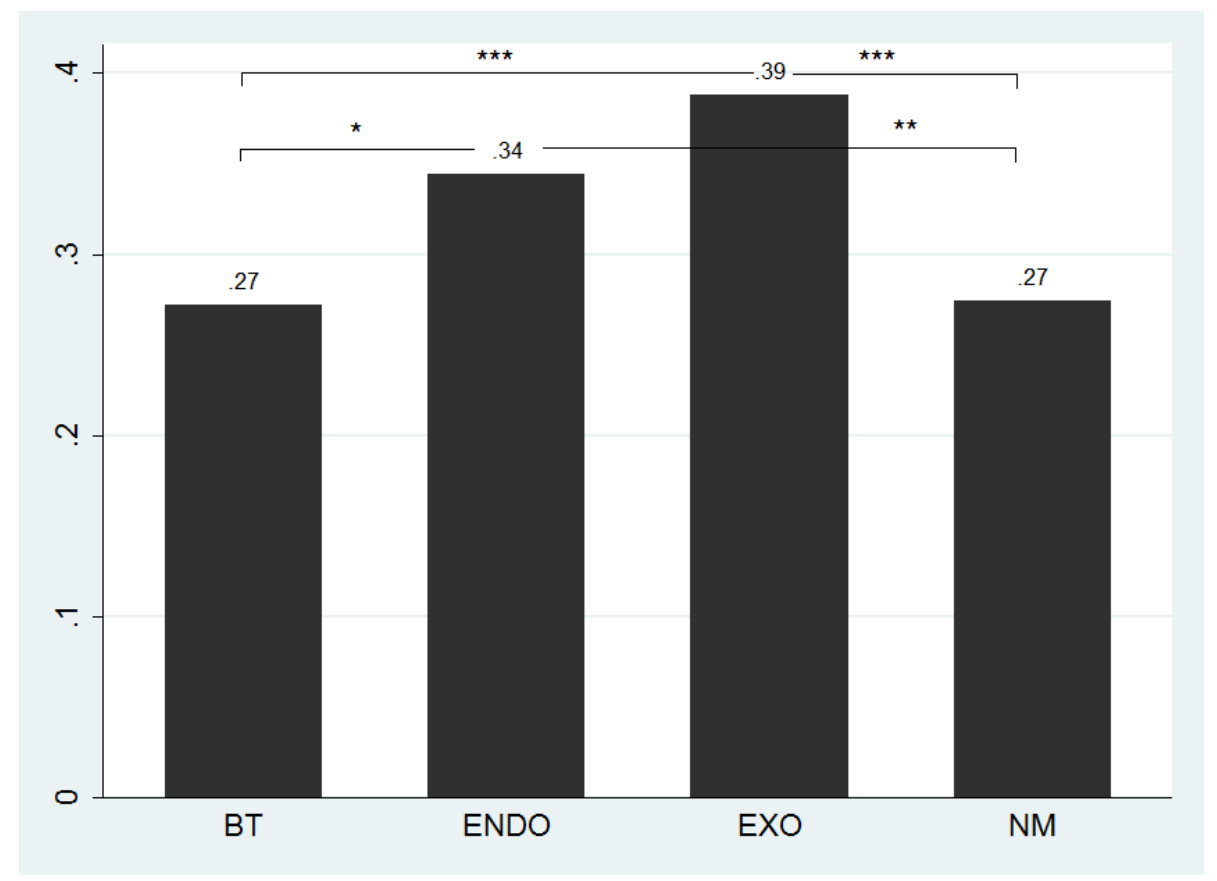

Note. In-degree centrality represents the asymmetry of network by taking the direction of links into account. The level of in-degree centrality is higher in EXO and in ENDO than in the two other treatments. ${ }^{*} \mathrm{p}<0.1$; ${ }^{* *} \mathrm{p}<0.05 ;{ }^{* * *} \mathrm{p}<0.01$.

$p=0.045$ between ENDO and NM and $p<0.001$ between EXO and BT and between EXO and NM.). However, the in-degree centrality levels are not significantly different between BT and $\mathrm{NM}(p=0.927)$.

Monetary heterogeneity facilitates the coordination of individuals on more central networks. We now focus on linking decisions of normal and special individuals to analyze what impacts the symmetry of networks in more details.

\subsection{Special vs. normal individuals' decisions}

Introducing heterogeneity has an effect on individual decision-making of special and normal individuals.

Result 3 (Attractiveness of special individuals) The special individuals in ENDO and EXO attract more links than normal individuals while the special individuals in NM are not. There is no significant difference between the results in ENDO and EXO.

Result 3 partly confirms the first part of Hypothesis 3. Special individuals in ENDO and EXO are more attractive than normal individuals, but not in NM. This result confirms the second part of Hypothesis 3, as the level of attractiveness of the special individual is higher in ENDO and EXO than in NM. It refutes the third part of Hypothesis 3 as there is no significant difference between ENDO and EXO. Normal individuals have an average in-degree ${ }^{15}$ per pe-

\footnotetext{
${ }^{15}$ Recall that the in-degree of individual $i$ is the number of links that individuals $j \neq i$ created with $i$.
} 
riod of 0.57 in ENDO and 0.51 in EXO, while the special individual has an average in-degree of 1.8 in ENDO and 1.79 in EXO. The difference in in-degree between normal and special individuals can be tested with a non-parametric test. We have 8 special individuals in each heterogeneous treatment. For each non-parametric tests on the decisions of individuals, we compare the means of decisions between normal and special individuals and the tests are twotailed. The difference of average in-degree is significant in ENDO and EXO between the special and the normal individuals $(p<0.001)$. Merit has no effect on the level of attractiveness. Indeed, there is no significant difference between the in-degree of special individuals in ENDO and $\operatorname{EXO}(p=0.779)$. The difference is also non significant for normal individuals $(p=0.165)$.

One reason of the fact that fairness levels are higher in ENDO and EXO is that the special individuals in ENDO and EXO can earn less than normal individuals because they can only be linked with normal individuals, while normal individuals can benefit from the higher value of special individuals. It is even possible that individuals understood that and created more links with the special individual to compensate and equalize payoffs.

Even if only five networks, over 160 formed in ENDO and EXO, are a PSS with the special individual as central agent, the additional monetary value of special individuals attracts normal individuals and increases the asymmetry of networks. The fact that groups only formed few PSS show that they understood that being indirectly connected to the special agent is sufficient to benefit from his higher value.

In NM, the special individual does not attract more links than normal individuals. Indeed, on average the special and normal individuals have an in-degree of 0.78 links during the experiment ( 0.79 for the special individual and 0.77 for normal individuals). The difference is not significant $(p=0.912)$. For the comparison, in BT, individuals have on average an in-degree of 0.79 . There is no significant difference between the number of links received by individuals in BT and in NM $(p=0.5217)$. Having the favorite profile in the group in terms of personal attributes and tastes is not sufficient to attract the other individuals. Figure 5 shows the average in-degree received by each type of individual in each treatment.

Special individuals in ENDO and EXO remain passive during the experiment. As they attract links, they rapidly know that they will be part of the network at the end of the period. Consequently, on average, special individuals in ENDO and EXO respectively remain passive in $80 \%$ and $90 \%$ of the time. This is not a significant difference, $p=0.116$. On the contrary, normal individuals in ENDO and EXO remained passive in only $13 \%$ of their decisions. As in NM special individuals do not attract more links than normal individuals, there is no significant difference between the level of passivity of normal and special individuals. Both types of individuals remain passive $24 \%$ of the time $(p=0.868)$. Moreover, the level of passivity is the same for individuals in NM and for individuals in BT $(p=0.868)$. The consequence of this result is that normal individuals create more links than special individuals in ENDO and EXO. They have to compensate the passivity of special individuals. Indeed normal individuals in ENDO and EXO create a link in $86.9 \%$ and $87.5 \%$ of the cases respectively, while normal individuals in BT and NM create a link in $76.5 \%$ and $75.9 \%$ of the cases. The difference is significant at the $1 \%$ level for each pairwise comparison between the monetary heterogeneous treatments and the two other treatments. 
Figure 5: In-degree of normal and special individuals

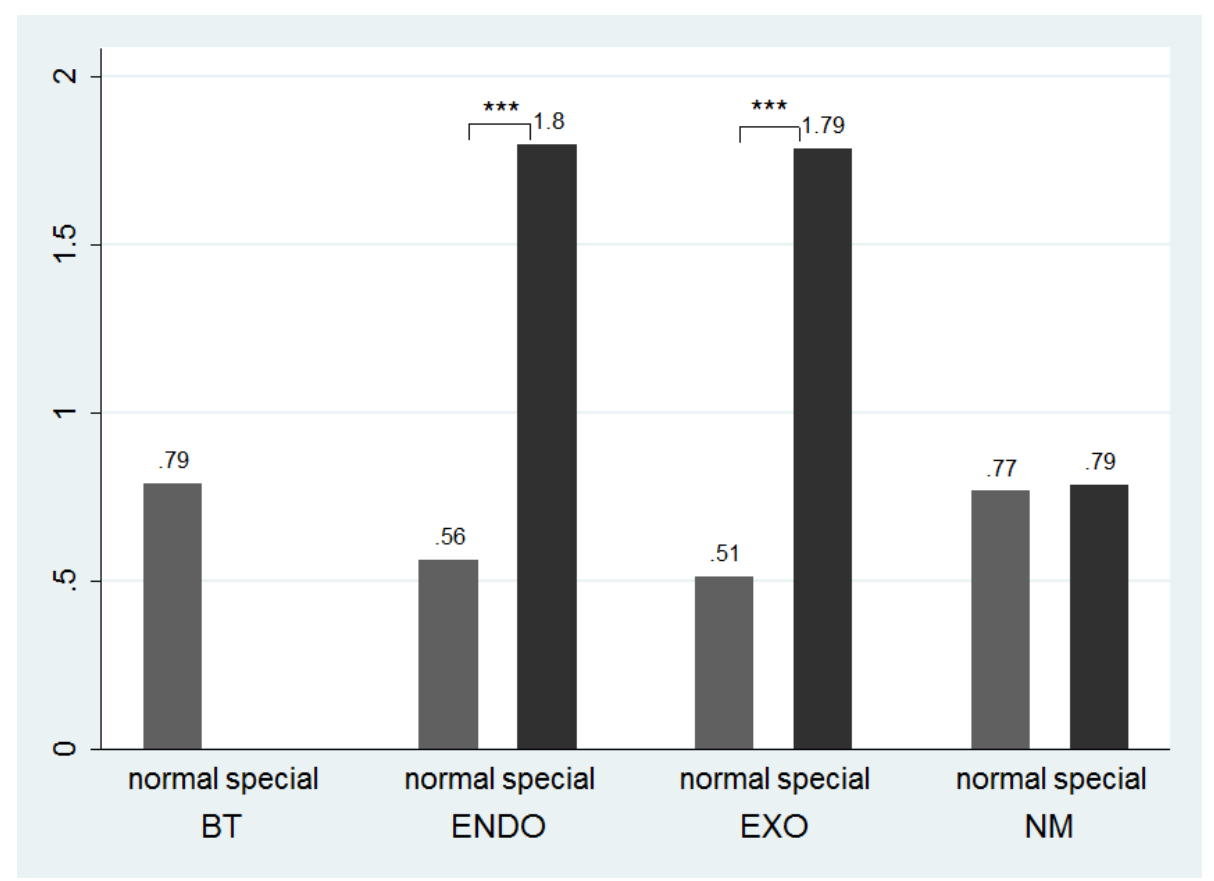

Note. The difference in in-degree (i.e. the in-degree of $i$ is the number of links that individuals $j \neq i$ created with $i)$ is significant between normal and special individuals in ENDO and EXO $(p<0.001)$ while it is not in NM $(p=0.912)$. The difference in in-degree for special and normal individuals is not significant between ENDO and EXO $(p>0.999)$. Finally, there is no significant difference between the in-degree of individuals between BT and NM $(p=0.5217) .{ }^{*} \mathrm{p}<0.1 ;{ }^{* *} \mathrm{p}<0.05 ;{ }^{* * *} \mathrm{p}<0.01$. 
More generally, when someone has already created a link with an individual, in $61.6 \%$ of the cases on average in all treatments, this individual decides not to create any link regardless of the decision order. However, there exists a significant difference between normal and special individuals in this case. When a normal individual has no existing link, he only remains passive in less than $10 \%$ of the decisions (BT: $5.68 \%$, ENDO: $7.81 \%$, EXO: $8.74 \%$ and NM: $7.55 \%$ ). Special individuals also remain passive in less than $10 \%$ of the time in NM $(5.66 \%)$ and BT $(5.68 \%)$ but a special individual in ENDO and EXO respectively remains passive in $87.5 \%$ and $80.77 \%$ of the time.

\subsection{First link created}

Result 4 (1st link created) The first link is created with the special individual in $59 \%$ of the cases in ENDO and $74 \%$ in EXO, which gives an insurance to be linked with the special individual.

In comparison with this result, only $22.5 \%$ of the first links are made with the special individual in NM. Note that when an individual faces an empty network of four individuals and creates the first link, if he chooses at random there is a $25 \%$ chance that he chooses to create a link with the special individual. Overall, there is a significant difference between the number of first links created with the special individual in the three heterogeneous treatments. The pairwise comparison of the average number of first links with the special individual shows that there is significantly more first links created with the special individual in ENDO and EXO than in NM ( $p<0.001$ for both). The difference is also significant between ENDO and EXO, $p=0.045$. Creating directly a link with the special individual can be seen as an insurance to be part of the network in ENDO and EXO. Forming immediately a link with this individual guarantees some benefits that are higher than when the individual connects to someone else and the individual knows that others will want to "join" them.

Another result can confirm this phenomenon. We studied the links created with the special individual over the decision order in the sequential process. Individuals who play first in the sequential process create more links with the special individuals than individuals who play later $\left(p<0.001\right.$ between the individual who play $1^{\text {st }}$ and the $2^{\text {nd }}, 3^{\text {rd }}$ and $5^{\text {th }}$ agent and $p=0.004$ with the $4^{t h}$ individual in the sequential process). When a link has been created with the special individual, individuals understand that they can create links with any individual of the component created.

This behavior is also related to risk aversion. When an individual plays first or has no links when it is his turn to decide, he faces two options: not creating any links or creating at least one. If he does not create any link, he exposes himself to others. Indeed, if he does not create any link, he cannot be sure that others will create a link with him. He can be excluded. When a special individual is present, a normal individual may prefer to create a link with him to be insured to be part of the same component than the special individual.

\subsection{Regression analysis}

In order to better understand the determinants of link formation, we estimate a probit model with clustered standard errors by group. The dependent variable is the binary decision to create at least one link in a period. The independent variables are the following. ENDO, EXO and $N M$ are dummy variables for the heterogeneous treatments. SpecialENDO, SpecialEXO 
and SpecialNM are dummy variables that take value 1 when the individual is the special agent in each heterogeneous treatment. The decision order of the individuals is given by the dummy variables Order 2-5. We study the effect of the order in the decision process to control for the fact that playing later in the network formation game increases the probability of being already linked with an individual. The Risk averse variable is a dummy variable given by the decision of individuals during the Gneezy and Potters (1997) test, where 0 represents both risk-lover and risk-neutral individuals and 1 represents risk-averse individuals. Prosocial is the outcome of the Murphy et al. (2011) test where 1 represents pro-social individuals and 0 represents the other types of individuals: competitive and individualist. $35 \%$ are pro-social while $65 \%$ are individualist and one individual is competitive. The variable Period 1 controls for the possible effect of the first period. We want to check whether agents behave differently at the first period as no networks have been formed before, so there is no learning effect. Finally, the Period 10 variable is a dummy variable for the $10^{\text {th }}$ period of the first session where we ran 15 periods instead of 10 . The 5 additional periods of this session are omitted.

We estimate two probit models (1) and (2). In the first model, the independent variables are the dummy variables ENDO, EXO and NM, the dummy variables Order 2-5 and the dummy variables Risk averse, Prosocial, Period 1 and Period 10. In the second model, we add the dummy variables SpecialENDO, SpecialEXO and SpecialNM to assess their impact on link formation. The marginal effects are depicted in Table 2.

Result 5 (First movers) Second movers are 16\% less likely to create links than first movers.

Playing later in the sequential process increases the likelihood of being already connected with another individual when it is one's turn to decide. Consequently, it decreases the likelihood to create a link. With the SpecialENDO, SpecialEXO and SpecialNM variables, we confirm our previous results and observe that being the special individual in ENDO and EXO significantly decreases the likelihood of creating a link while it has no significant effect in NM. On the contrary, normal individuals in these treatments have to compensate by creating more links. This is reflected by the positive and significant effect of ENDO and EXO in model (2).

The Risk averse and Prosocial variables do not significantly impact link creation. Finally, we observe no effect of Period 1 nor of Period 10 on linking decision. Thanks to the sequential process, the process of network formation is stable over time as well as the individual decision to create a link.

\section{Conclusion}

Asymmetric networks are prominent in real-life settings. However, in the lab, due to fairness concerns, they do not emerge. A feature that can explain this fact is that individuals have different characteristics that are not represented in the lab. Even if our theoretical model predicts the formation of a highly unfair and asymmetric network - the Center-Sponsored Star is the Subgame Perfect Equilibrium - the networks formed in our sequential network formation game have a high level of fairness as individuals tend to create one link each. This is due to the sequential process of link formation that allows groups to coordinate on fair and efficient networks and to build very stable networks. However, when we introduce heterogeneity via the presence of a special individual with a higher monetary value, the asymmetry of networks increase. The in-degree centrality of networks is higher in treatments with heterogeneity if this 
heterogeneity is monetary. In other words, the special individuals are more attractive than normal individuals in ENDO and EXO. Normal individuals tend to create more links with the special individuals than with any other individual. Nevertheless, non-monetary heterogeneity is not sufficient to lead to the attractiveness of the special individual. Our results show that being linked to the special individual in ENDO and EXO is an insurance to have a high payoff.

Our paper shows that heterogeneity is one of the determinants of the asymmetry of networks in real life. However, if we compare our results to the results in the paper of GOE, centrality levels are much higher in the latter. It seems that the decay assumption in GOE allows to increase the centrality of networks as the special individual becomes very attractive. Consequently, in real-life settings, the impact of heterogeneity can be more or less strong. For example, in a company network, having a valuable supplier may benefit all the network. On the contrary, in the scientific co-authorship network, it is very valuable to write a paper with a well-known author while being indirectly linked with him does not really impact one's payoff. Additional experimental works on network formation and the effect of heterogeneity are necessary to better understand its impact on the structure of networks.

\section{References}

Bala, V. and S. Goyal (2000). A noncooperative model of network formation. Econometrica 68(5), 1181-1229.

Ball, S. B. and C. C. Eckel (1998). Stars upon thars: status and discrimination in ultimatum games.

Ball, S. B., C. C. Eckel, P. J. Grossman, and W. Zame (2001). Status in markets. Quarterly Journal of Economics 116(1), 161-188.

Barabási, A.-L., R. Albert, and H. Jeong (2000). Scale-free characteristics of random networks: the topology of the world-wide web. Physica A: Statistical Mechanics and its Applications 281(1), 69-77.

Bernasconi, M. and M. Galizzi (2005). Coordination in networks formation: Experimental evidence on learning and salience.

Berninghaus, S. K., K.-M. Ehrhart, and M. Ott (2006). A network experiment in continuous time: The influence of link costs. Experimental Economics 9(3), 237-251.

Berninghaus, S. K., K.-M. Ehrhart, M. Ott, and B. Vogt (2007). Evolution of networks-an experimental analysis. Journal of Evolutionary Economics 17(3), 317-347.

Berninghaus, S. K., M. Ott, and B. Vogt (2004). On networks and stars: Recent results in network experiments.

Bock, O., I. Baetge, and A. Nicklisch (2014). Hroot: Hamburg registration and organization online tool. European Economic Review 71, 117-120.

Bramoullé, Y. and B. W. Rogers (2009). Diversity and popularity in social networks.

Buechel, B. and T. Hellmann (2012). Under-connected and over-connected networks: the role of externalities in strategic network formation. Review of Economic Design 16(1), 71-87. 
Callander, S. and C. R. Plott (2005). Principles of network development and evolution: An experimental study. Journal of Public Economics 89(8), 1469-1495.

Cherry, T. L., P. Frykblom, and J. F. Shogren (2002). Hardnose the dictator. The American Economic Review 92(4), 1218-1221.

Eckel, C. C. and R. K. Wilson (2007). Social learning in coordination games: does status matter? Experimental Economics 10(3), 317-329.

Falk, A. and M. Kosfeld (2012). It's all about connections: Evidence on network formation. Review of Network Economics 11(3).

Fehr, E. and K. M. Schmidt (1999). A theory of fairness, competition, and cooperation. Quarterly journal of Economics, 817-868.

Freeman, L. C. (1978). Centrality in social networks conceptual clarification. Social networks 1(3), 215-239.

Galeotti, A. and S. Goyal (2010). The law of the few. The American Economic Review, $1468-1492$.

Galeotti, A., S. Goyal, and J. Kamphorst (2006). Network formation with heterogeneous players. Games and Economic Behavior 54(2), 353-372.

Galeotti, F. and D. J. Zizzo (2014). What happens if you single out? an experiment. Social Choice and Welfare 43(3), 703-729.

Gill, D. and V. Prowse (2012). A structural analysis of disappointment aversion in a real effort competition. The American economic review, 469-503.

Gneezy, U. and J. Potters (1997). An experiment on risk taking and evaluation periods. The Quarterly Journal of Economics, 631-645.

Goeree, J. K., A. Riedl, and A. Ule (2009). In search of stars: Network formation among heterogeneous agents. Games and Economic Behavior 67(2), 445-466.

Goyal, S. and S. Joshi (2006). Unequal connections. International Journal of Game Theory $34(3), 319-349$.

Heffetz, O. and R. H. Frank (2008). Preferences for status: Evidence and economic implications. Handbook of social economics, Jess Benhabib, Alberto Bisin, Matthew Jackson, eds 1, 69-91.

Herings, J.-J. P., A. Mauleon, and V. Vannetelbosch (2009). Farsightedly stable networks. Games and Economic Behavior 67(2), 526-541.

Hoffman, E. and M. L. Spitzer (1985). Entitlements, rights, and fairness: An experimental examination of subjects' concepts of distributive justice. The Journal of Legal Studies 14(2), 259-297.

Karakayali, N. (2009). Social distance and affective orientations1. In Sociological Forum, Volume 24, pp. 538-562. Wiley Online Library. 
Mantovani, M., G. Kirchsteiger, A. Mauleon, and V. J. Vannetelbosch (2011). Myopic or farsighted? an experiment on network formation.

Morbitzer, D., V. Buskens, S. Rosenkranz, and W. Raub (2014). How farsightedness affects network formation. Analyse \& Kritik 36(1).

Morrill, T. (2011). Network formation under negative degree-based externalities. International Journal of Game Theory 40(2), 367-385.

Murphy, R. O., K. A. Ackermann, and M. Handgraaf (2011). Measuring social value orientation. Judgment and Decision Making 6(8), 771-781.

Newman, M. E. (2004). Who is the best connected scientist? a study of scientific coauthorship networks. In Complex networks, pp. 337-370. Springer.

Riedl, A. and A. Ule (2002). Exclusion and cooperation in social network experiments.

Rong, R. and D. Houser (2012). Growing stars: A laboratory analysis of network formation.

Rosenkranz, S. and U. Weitzel (2012). Network structure and strategic investments: An experimental analysis. Games and Economic Behavior 75(2), 898-920.

Van Dolder, D. and V. Buskens (2014). Individual choices in dynamic networks: An experiment on social preferences. PloS one 9(4), e92276.

Van Leeuwen, B., T. Offerman, and A. J. Schram (2015). Competition for status creates superstars: An experiment on public good provision and network formation.

Vanin, P. (2002). Network formation in the lab: a pilot experiment.

Weiss, Y. and C. Fershtman (1998). Social status and economic performance:: A survey. European Economic Review 42(3), 801-820.

\section{Appendix}


Figure 6: Screenshot of treatments ENDO and EXO

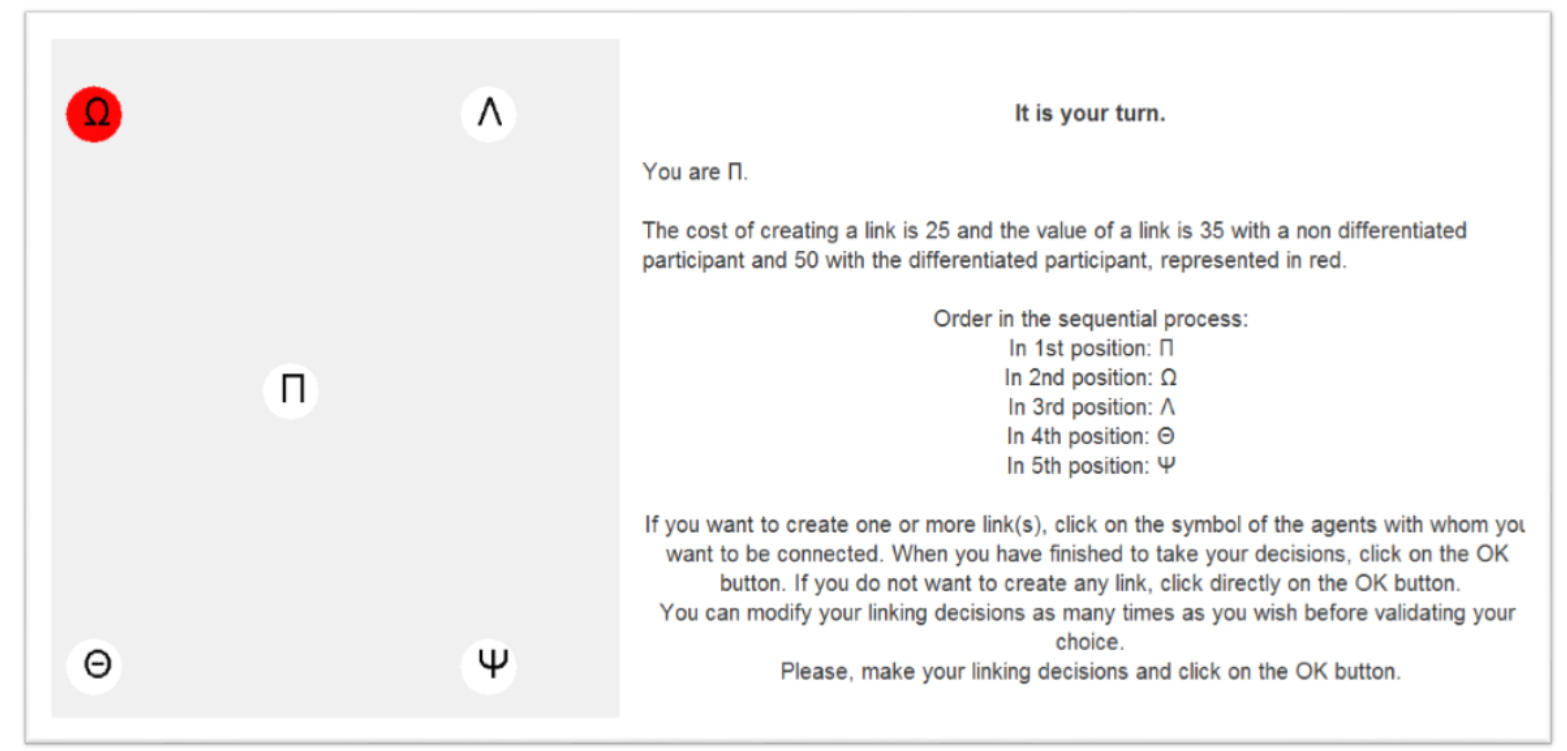

Figure 7: Screenshot of treatment NM

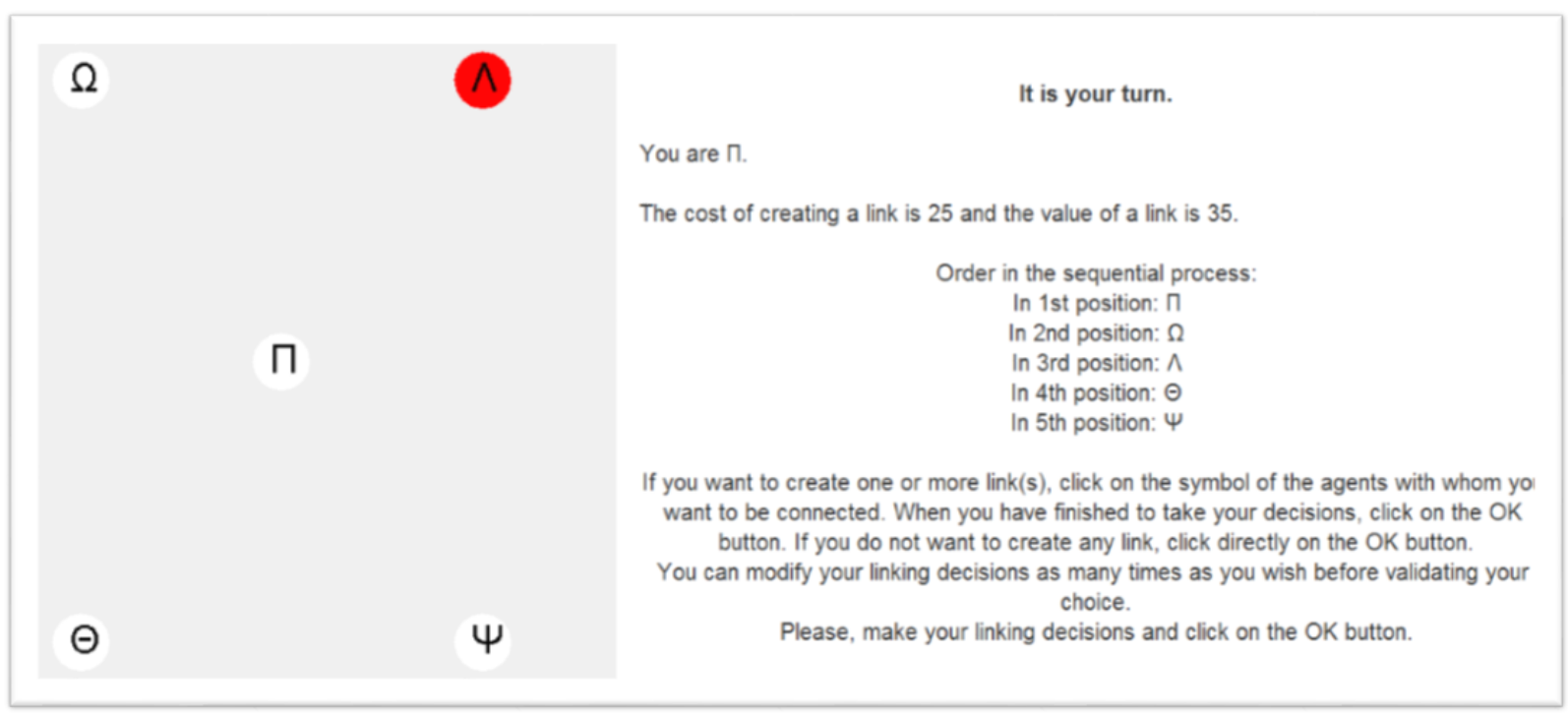


Table 2: Marginal effects, probit, clustered standard errors at the group level

\begin{tabular}{|c|c|c|}
\hline & \multicolumn{2}{|c|}{ Decision to create link(s) } \\
\hline ENDO & $\begin{array}{l}-0.027 \\
(0.036)\end{array}$ & $\begin{array}{c}0.118^{* *} \\
(0.047)\end{array}$ \\
\hline $\mathrm{EXO}$ & $\begin{array}{c}-0.052 \\
(0.036)\end{array}$ & $\begin{array}{l}0.121^{* *} \\
(0.045)\end{array}$ \\
\hline NM & $\begin{array}{c}0.006 \\
(0.023)\end{array}$ & $\begin{array}{c}0.004 \\
(0.028)\end{array}$ \\
\hline SpecialENDO & - & $\begin{array}{c}-0.677^{* * *} \\
(0.053)\end{array}$ \\
\hline SpecialEXO & - & $\begin{array}{c}-0.754^{* * *} \\
(0.041)\end{array}$ \\
\hline SpecialNM & - & $\begin{array}{c}0.005 \\
(0.058)\end{array}$ \\
\hline Order 2 & $\begin{array}{c}-0.107^{* * *} \\
(0.043)\end{array}$ & $\begin{array}{c}-0.163^{* * *} \\
(0.041)\end{array}$ \\
\hline Order 3 & $\begin{array}{c}-0.132^{* * *} \\
(0.049)\end{array}$ & $\begin{array}{c}-0.192^{* * *} \\
(0.052)\end{array}$ \\
\hline Order 4 & $\begin{array}{c}-0.087^{*} \\
(0.053)\end{array}$ & $\begin{array}{c}-0.135^{* *} \\
(0.06)\end{array}$ \\
\hline Order 5 & $\begin{array}{c}-0.093^{*} \\
(0.052)\end{array}$ & $\begin{array}{c}-0.147^{* * *} \\
(0.054)\end{array}$ \\
\hline Risk averse & $\begin{array}{c}0.065 \\
(0.042)\end{array}$ & $\begin{array}{c}0.055 \\
(0.035)\end{array}$ \\
\hline Prosocial & $\begin{array}{c}0.038 \\
(0.039)\end{array}$ & $\begin{array}{c}0.021 \\
(0.023)\end{array}$ \\
\hline Period 1 & $\begin{array}{l}0.007 \\
(0.02)\end{array}$ & $\begin{array}{c}0.009 \\
(0.025)\end{array}$ \\
\hline Period 10 & $\begin{array}{c}0.096 \\
(0.061) \\
\end{array}$ & $\begin{array}{c}0.094 \\
(0.057) \\
\end{array}$ \\
\hline Observations & 1600 & 1600 \\
\hline Pseudo $r^{2}$ & 0.014 & 0.194 \\
\hline
\end{tabular}

Note: ${ }^{*} p<0.1$; $^{* *} p<0.05$; $^{* *} p<0.01$. 